\title{
NEAR-INFRARED PHOTOMETRY OF THE GLOBULAR CLUSTER PALOMAR 6
}

\author{
JAe-Woo LeE ${ }^{1,2,3}$ AND Bruce W. CARneY ${ }^{1,4}$ \\ Received 2001 September 19; accepted 2002 February 28
}

\begin{abstract}
We present $J H K$ photometry of Palomar 6. Our photometric measurements range from the red giant branch (RGB) tip to $\approx 2$ mag below the red horizontal branch (RHB), and our color-magnitude diagrams show that Palomar 6 appears to have a well-defined RHB population. The distance modulus and interstellar reddening of the cluster are estimated by comparing the magnitude and color of Palomar 6 RHB stars with those of 47 Tuc. We obtain $(m-M)_{0}=14.28 \mathrm{mag}$ and $E(B-V)=1.30 \mathrm{mag}$ for the cluster, and our study suggests that Palomar 6 is clearly located in the Galaxy's central regions. We also discuss the metallicity of the cluster using the slope of the RGB. We obtain $[\mathrm{Fe} / \mathrm{H}] \approx-1.2$ for Palomar 6 and our metallicity estimate is $\approx 0.5-1.0$ dex lower than previous estimates by others.
\end{abstract}

Key words: color-magnitude diagrams — globular clusters: individual (Palomar 6) stars: horizontal-branch — stars: Population II

On-line material: machine-readable table

\section{INTRODUCTION}

Palomar $6\left(\alpha=17^{\mathrm{h}} 44^{\mathrm{m}}, \delta=-26^{\circ} 13^{\prime} ; l=2.1, b=1.8\right.$, $\mathrm{J} 2000.0$ ) is a globular cluster $\approx 0.8 \mathrm{kpc}$ from the Galactic center and $\approx 0.2 \mathrm{kpc}$ from the plane (Harris 1996). Since it lies near the Galactic center, it is highly reddened.

Malkan (1981) derived the interstellar reddening for Palomar $6, E(B-V)=1.4$, based on a reddening-free metallicity index $^{5} Q_{\text {IR. }}$. Ortolani, Bica, \& Barbuy (1995) obtained $E(B-V)=1.33 \pm 0.10$, based on the $V I$ color-magnitude diagram (CMD). Bica et al. (1998) also studied the cluster, employing intermediate-resolution $\left(5.4 \AA\right.$ pixel $\left.^{-1}\right)$ integrated spectra, and they derived the smallest value of the interstellar reddening value for the cluster, $E(B-V)=1.25$.

The metallicity of the cluster is rather controversial. Malkan (1981) obtained a metallicity of $[\mathrm{Fe} / \mathrm{H}]=-1.30$ for Palomar 6 by using the $Q_{\mathrm{IR}}$ index, ranking it as an intermediate-metallicity inner halo cluster. Zinn (1985) derived $[\mathrm{Fe} / \mathrm{H}]=-0.74$ for Palomar 6 by reanalyzing Malkan's photometry. Ortolani et al. (1995) estimated $[\mathrm{Fe} / \mathrm{H}] \approx$ -0.4 , based on the VI CMD. Minniti (1995) studied the metallicities of seven reddened clusters including Palomar 6. He obtained spectra of six red giant branch (RGB) stars in Palomar 6 with a $2 \AA$ resolution covering 4700-5400 $\AA$. Following Faber et al. (1985), he suggested that the sum of the $\mathrm{Mg} 5175, \mathrm{Fe} 5270$, and Fe $5535 \mathrm{~A}$ lines (Mg and 2Fe) is the most sensitive to metallicity, and he derived

${ }^{1}$ Department of Physics and Astronomy, University of North Carolina, Chapel Hill, NC 27599-3255; jaewoo@astro.unc.edu, bruce@ physics.unc.edu.

${ }^{2}$ Center for Space Astrophysics, Yonsei University, Shinchon-dong 134, Seoul 120-749, Korea.

${ }^{3}$ PMA Division, Mail Stop 405-47, California Institute of Technology, Pasadena, CA 91125; jaewoo@srl.caltech.edu.

${ }^{4}$ Visiting Astronomer, Kitt Peak National Observatory, National Optical Astronomy Observatory, which is operated by the Association of Universities for Research in Astronomy, Inc., under cooperative agreement with the National Science Foundation.

${ }^{5} Q_{\mathrm{IR}}$ is defined as $\mathrm{H}_{2} \mathrm{O}+1.5 \mathrm{CO}$, where $\mathrm{H}_{2} \mathrm{O}$ and $\mathrm{CO}$ are the narrowband infrared photometric absorption indices from $\mathrm{H}_{2} \mathrm{O}$ at $2.0 \mu \mathrm{m}$ and $\mathrm{CO}$ at $2.4 \mu \mathrm{m}$. Malkan (1981) claimed that there is a correlation between $Q_{\mathrm{IR}}$ and a reddening-free ultraviolet line-blanketing parameter $Q_{39}$ (Zinn 1980) with $Q_{\mathrm{IR}}=0.045+0.45 Q_{39}$.
$[\mathrm{Fe} / \mathrm{H}]=+0.2 \pm 0.3$ for Palomar 6 based on the location of the giants in the $\mathrm{Mg}+2 \mathrm{Fe}$ versus $(J-K)_{0}$ diagram. More recently, Bica et al. (1998) obtained $Z / Z_{\odot}=-0.09$ by measuring the $\mathrm{Ca}$ II triplet $\lambda \lambda 8498,8542$, and 8662 by using the intermediate-resolution integrated spectra.

As discussed above, Palomar 6 suffers a large interstellar reddening. The interstellar extinction in the visual passband $A_{V}$ for Palomar 6 is expected to be $\approx 3.9-4.3 \mathrm{mag}$, depending on its true interstellar reddening value, and the apparent visual magnitude of the horizontal branch (HB) $V_{\mathrm{HB}}$ is $\approx 20$ mag (Ortolani et al. 1995). Thus, photometric study of Palomar 6 in the visual passband is very difficult. It is also likely that differential reddening, which is commonly detected in heavily reddened clusters, is present. The IR photometry is, therefore, essential for studying Palomar 6, since it is less sensitive to the interstellar reddening and the differential reddening effect. In this paper, we explore the ground-based $J H K$ photometry of Palomar 6. A comparison with $J K$ photometry of Minniti, Olszewski, \& Rieke (1995) is made. The distance modulus using the $K$ magnitude of the red horizontal branch (RHB) and the metallicity estimate using the RGB slope of the cluster are discussed.

\section{OBSERVATIONS AND IR DATA REDUCTION TECHNIQUES}

Our observations were carried out using the $4 \mathrm{~m}$ telescope at KPNO on the night of 1997 June 15 under photometric conditions. The detector was a $256 \times 256 \mathrm{HgCdTe}$ NICMOS3 array and the $J H K$ filter system was employed. The image scale was 0 .'60 pixel $^{-1}$, providing a field of view (FOV) of $2.56 \times 2 ! 56$. The journal of observations is presented in Table 1.

During our observations, we dithered the telescope pointing to minimize the effects induced by bad pixels and cosmic-ray events. For standard-star frames, the telescope pointing was dithered with an offset of $37^{\prime \prime}$ between successive exposures. Offsets of $6^{\prime \prime}$ and $10^{\prime \prime}$ were applied for Palomar 6 object frames.

NICMOS3 detectors utilize a hybrid architecture in which each pixel has an associated unit cell, which controls 
TABLE 1

JOURNAL OF OBSERVATIONS

\begin{tabular}{cccc}
\hline \hline Band & $\begin{array}{c}t_{\exp } \\
(\mathrm{s})\end{array}$ & $\begin{array}{c}\text { FWHM } \\
(\operatorname{arcsec})\end{array}$ & $\begin{array}{c}\text { Dither } \\
(\operatorname{arcsec})\end{array}$ \\
\hline$J \ldots \ldots \ldots$ & $0.5 \times 5$ & 1.0 & 6,10 \\
$J \ldots \ldots \ldots$. & $5.0 \times 5$ & 1.0 & 6,10 \\
$H \ldots \ldots \ldots$ & $0.5 \times 5$ & 1.0 & 6,10 \\
$H \ldots \ldots \ldots$ & $5.0 \times 5$ & 1.0 & 6,10 \\
$K \ldots \ldots \ldots$ & $0.5 \times 5$ & 1.0 & 6,10 \\
$K \ldots \ldots \ldots$. & $5.0 \times 5$ & 1.0 & 6,10 \\
\hline
\end{tabular}

the biasing and readout of the pixel. Thus, each pixel is essentially independent of the others, and charge bleeding or trailing from saturated pixels is not present. However, this independence also means that such properties as linearity and dark current can vary from pixel to pixel, and it is necessary to calibrate for these effects for optimum scientific performance.

The mean dark current of NICMOS3 is known to be $\approx 2$ $e^{-} \mathrm{s}^{-1}$. However, it does not scale simply with the exposure time. Thus we obtained dark frames with the same exposure times as object and standard-star frames to calibrate the dark currents.

The nonlinearity of the detector sensitivity was also calibrated. We obtained a series of dome flats with exposure times of $3,10,15,20$, and $30 \mathrm{~s}$, maintaining the same illumination intensity. We also obtained $1 \mathrm{~s}$ exposures before and after each exposure to monitor possible changes in the illumination. Then the fluxes of each exposure were normalized to that of $1 \mathrm{~s}$ exposures. The measured fluxes for each exposure were plotted as a function of expected fluxes with linear sensitivity. Then we derived a third-order relation,

$$
\begin{aligned}
\mathrm{ADU}_{\text {corr }}= & \mathrm{ADU}_{\mathrm{obs}}\left[0.9952+0.0744 \frac{\mathrm{ADU}_{\mathrm{obs}}}{32767}\right. \\
& \left.-0.0489\left(\frac{\mathrm{ADU}_{\text {obs }}}{32767}\right)^{2}\right]
\end{aligned}
$$

where $\mathrm{ADU}_{\text {corr }}$ and $\mathrm{ADU}_{\mathrm{obs}}$ are the corrected flux and the observed flux in ADU, respectively. This relation was then applied to each object frame using the $\mathrm{IRAF}^{6}$ task IRLINCOR. Figure 1 shows the nonlinear response curve of the NICMOS3 detector. The solid line represents the linear relation, filled circles represent the observed fluxes, and open circles represent the corrected fluxes using the relation above.

The sky flats were generated by median-combining object frames of the individual dithering sequences for each passband. This procedure essentially removes the stellar light and cosmic rays and provides good sky flats for less crowded fields, such as standard-star frames. The sky flats generated using Palomar 6 object frames, however, show residuals of stellar images, indicating that this procedure fails when the field is too crowded. Applying sky flats generated from the other images taken at different times may cause problems. It is known that there are two predominant

${ }^{6}$ IRAF (Image Reduction and Analysis Facility) is distributed by the National Optical Astronomy Observatory, which is operated by the Association of Universities for Research in Astronomy, Inc., under contract with the National Science Foundation.

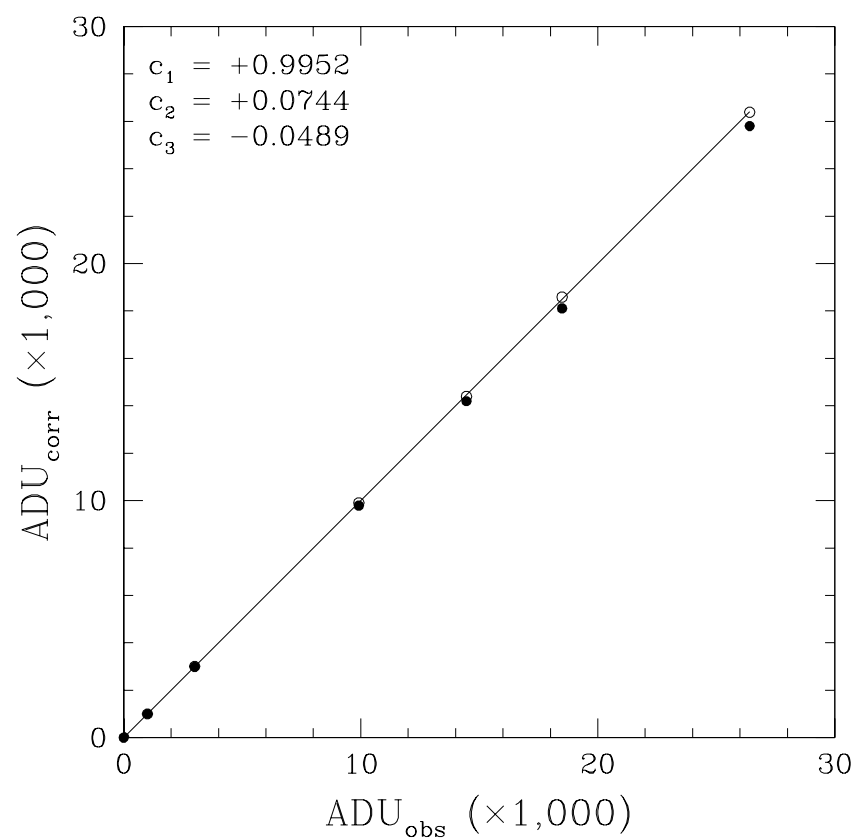

FIG. 1.-Nonlinear response of NICMOS3 detector, showing the observed fluxes ( filled circles), the corrected flux in ADU (open circles), and the linear response function (solid line).

sources of infrared sky background, which are essentially independent, both physically and spectrally. At short wavelengths (in particular $H$ passband), the sky is dominated by emission lines from $\mathrm{OH}$ in the upper atmosphere (typically $90 \mathrm{~km}$ altitude). The strength of these lines can vary over the course of a night; in addition, upper level winds create inhomogeneity and motion of the airglow. As a result, the intensity of the background can vary unpredictably during the night. At longer wavelengths, thermal emission from the telescope optics and optically thick telluric lines predominates. The transition between these two regimes occurs at approximately $2.3 \mu \mathrm{m}$, so the background with filters other than $K^{\prime}$ or $K$ is primarily $\mathrm{OH}$ airglow. We produced master sky flats, which were median-combined sky flats of the whole set of standard-star frames, rather than using sky flats from a single dithering sequence. Since the standard-star frames were taken over the night in our observation, using these master sky flats will minimize the risk of the abrupt changes of infrared sky background.

Figure 2 shows a comparison between the sky flat generated using the Palomar 6 frame (upper left) and our master sky flat (upper right) in the $J$ passband. As can be seen, the sky flat using the Palomar 6 frames shows residuals of stellar light. This causes serious problems in the central part of the cluster, where the field is more crowded. The flat-field corrected images are also shown in the figure. Figure 2 (lower left) shows the final image using the sky flat from the Palomar 6 frames, and Figure 2 (lower right) shows that using the master sky flat. The former does not resolve fainter stars very well, and even brighter stars turned out to be affected by the residual flux on the sky flat in the central part of the cluster. Therefore, we used the master sky flat in our analysis.

It is known that there are several hundred bad pixels in the NICMOS3 detector used in our observation. The badpixel correction was applied after the flat-field correction 

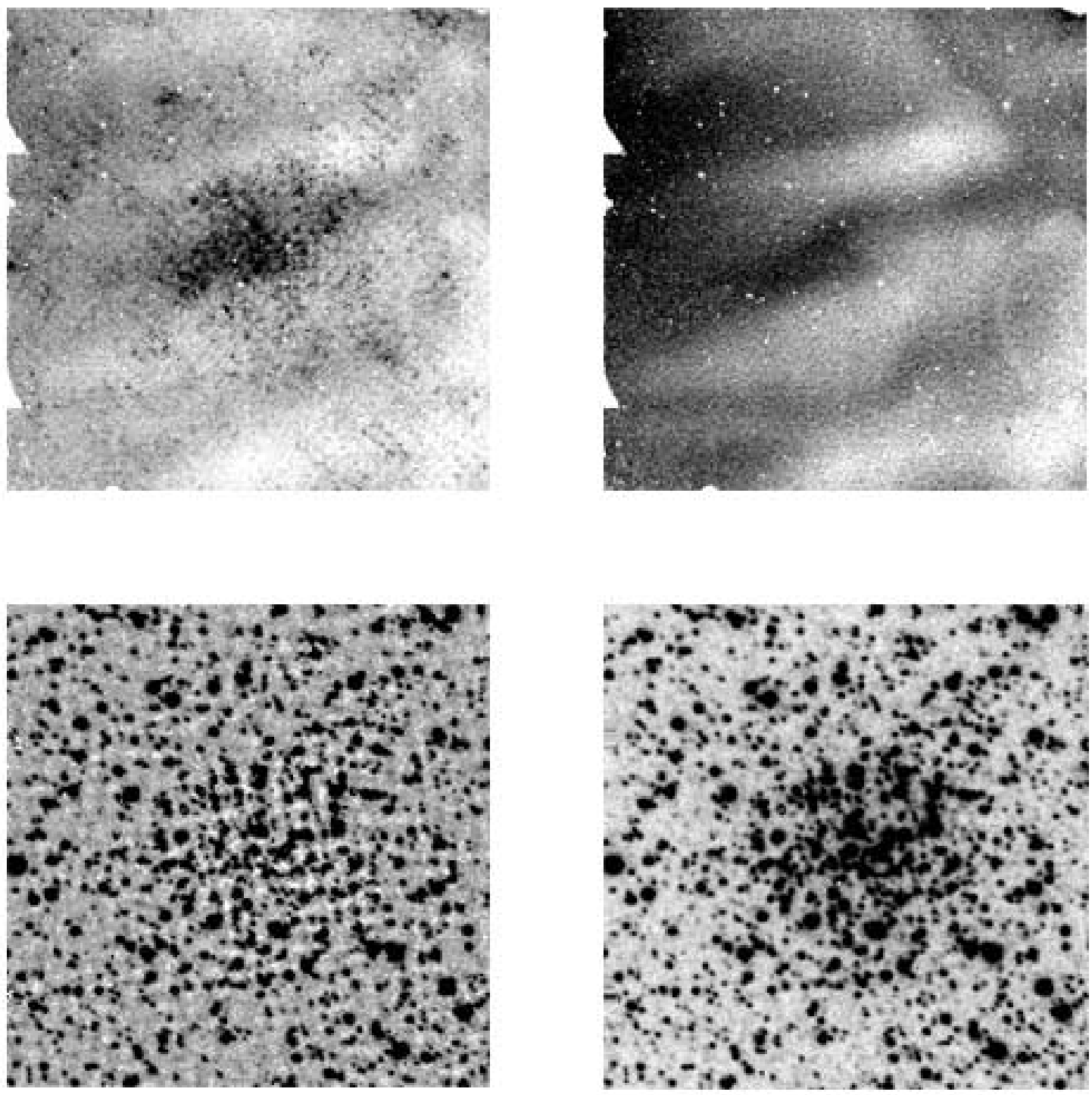

Fig. 2.-Top left, sky flat generated from the Palomar 6 object frames, showing the residual light from stellar images especially in the central part of the frame; top right, master sky flat generated from the standard-star frames; bottom, flat-field corrected images for each case.

step. The bad-pixel mask was generated using the standardstar frames. The flat-field corrected standard-star frames $(\approx 100$ frames) were normalized and then median combined. Since the strict definition of a bad pixel is rather subjective except for the nonresponsive (dead) pixels, the pixels deviating more than $7 \sigma$ around the mean value (1.0) were defined as bad pixels. The pixel values at the bad-pixel positions were interpolated using those of nearby pixels. Finally, the object frames in each dithering sequence were combined. Our combined image of Palomar 6 in $K$ passband is shown in Figure 3.

During the night, we observed the six UKIRT faint standard stars (Casali \& Hawarden 1992). It should be noted that the number of IR standard stars is small and most of the IR standard stars by Elias et al. (1982) are too bright for our camera and telescope configuration. All standard frames were analyzed using the PHOTOMETRY task in DAOPHOTII (Stetson 1995). With the results of aperture photometry, growth curve analysis was performed using DAOGROW (Stetson 1990) to obtain integrated magnitudes.
The extinction coefficients were derived from one standard star (FS 25). The first-order $K$ passband extinction term $k_{k}$ is 0.101 mag air mass ${ }^{-1}$, and the first-order color extinction terms $k_{j k}^{\prime}$ and $k_{j h}^{\prime}$ are -0.004 and 0.045 mag air mass ${ }^{-1}$, respectively.

To calibrate the photometry, the photometric transformations were assumed to have the following form:

$$
\begin{aligned}
K_{\mathrm{UKIRT}} & =k_{0}+\zeta_{K}, \\
(J-K)_{\mathrm{UKIRT}} & =\mu(j-k)_{0}+\zeta_{J-K}, \\
(J-H)_{\mathrm{UKIRT}} & =\epsilon(j-h)_{0}+\zeta_{J-H} .
\end{aligned}
$$

The transformation coefficients are $\zeta_{\mathrm{K}}=-3.235 \pm 0.010$, $\mu=0.957 \pm 0.019, \quad \zeta_{J-K}=0.624 \pm 0.007, \quad \epsilon=1.006$ \pm 0.016 , and $\zeta_{J-H}=0.156 \pm 0.004$ in the UKIRT (UK) $J H K$ system. (The errors are those of the mean.) Figure 4 shows the residuals of $K_{\mathrm{UK}},(J-K)_{\mathrm{UK}}$, and $(H-K)_{\mathrm{UK}}$, using these transformation coefficients. The mean values are $0.000 \pm 0.010$ in the $K_{\mathrm{UK}}$ passband, $0.000 \pm 0.010$ in the $(J-K)_{\mathrm{UK}}$ color, and $0.000 \pm 0.007$ in the $(H-K)_{\mathrm{UK}}$ color. (The errors are those of the mean.) 


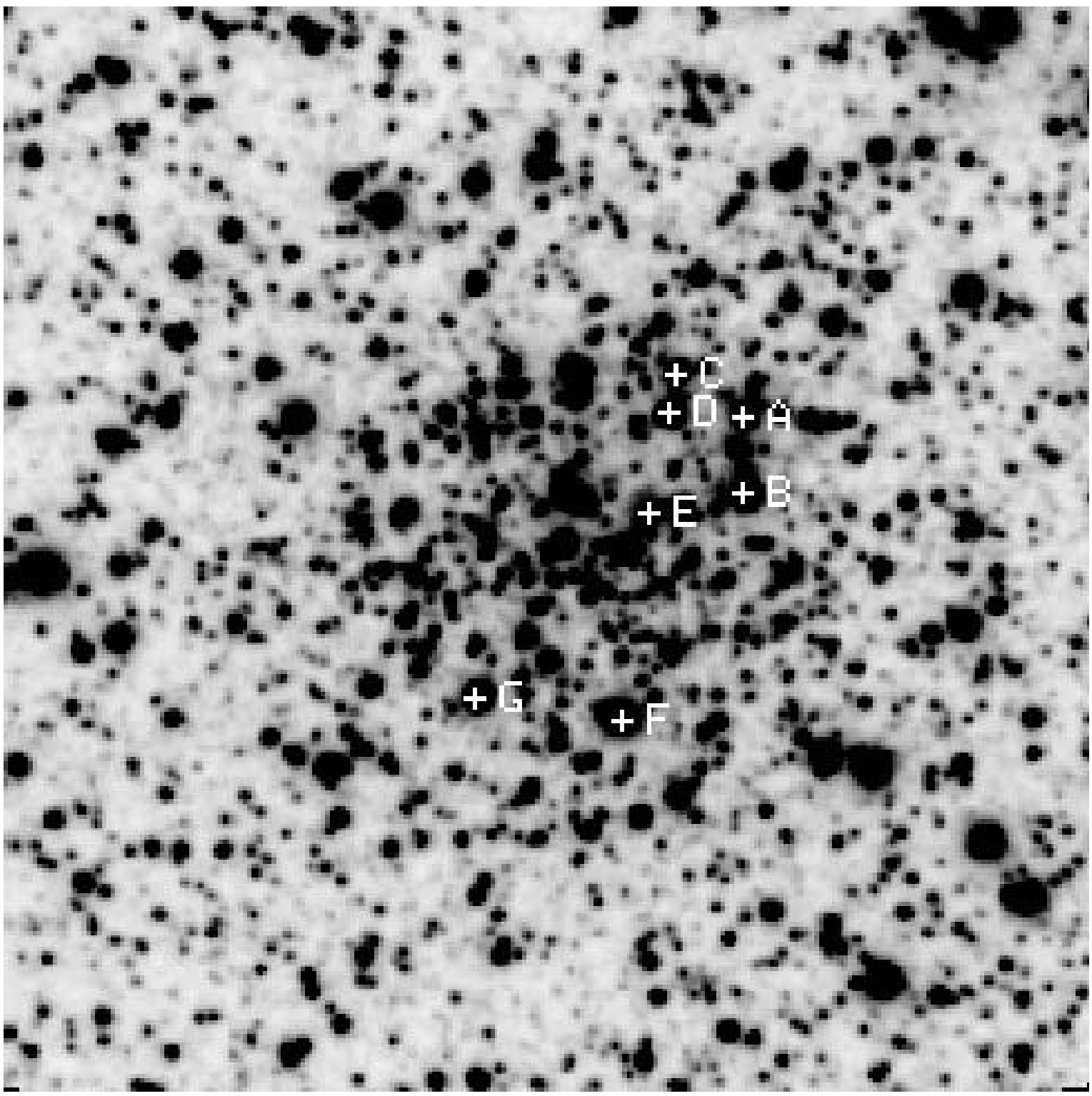

FIG. 3.-Composite image of Palomar 6 in $K$ passband. North is at the top, and east is to the left. The RGB stars with known heliocentric radial velocities in Table 4 are also marked.

Point-spread function (PSF) photometry for all Palomar 6 frames was performed using DAOPHOTII and ALLSTAR/ALLFRAME (Stetson 1987, 1994, 1995; Turner 1995). The first step was to perform the aperture photometry with star lists returned from the FIND routine. We calculated PSFs by using isolated bright stars. To obtain good PSFs on each frame, at least three iterations of neighboring star removal were performed. For the PSF calculations, we adopted a spatially invariable PSF since, unlike modern CCDs, a NICMOS3 array has a small FOV, so that the stellar radial profiles are not expected to show large variations over the frame. Also, the number of PSF stars is too small (usually 20-30 stars) to calculate reliable variable PSFs in each frame. In the case of the $2 \mathrm{~K}$ CCD system, the number of PSF stars is large enough (for example 90-110 stars in Lee \& Carney 1999) to calculate a variable PSF in each frame. After the first ALLFRAME pass, we undertook an additional pass to find more stars on the subtracted frames. Since we used a small PSF radius (usually $\approx 4 \times \mathrm{FWHM}$ ) to compute the PSF magnitude due to crowding, a PSF magnitude must be converted into an aperture magnitude with a larger radius, a process known as aperture correction. The 20 to $30 \mathrm{PSF}$ stars on each frame were used for this purpose. Comparisons of the PSF magnitude and the aperture magnitude from the growth curve method yielded aperture corrections. Finally air-mass corrections and photometric transformations were applied.

To convert the UKIRT $J H K$ system to the CIT $J H K$ system, we used the transformation equations

$$
\begin{aligned}
K & =K_{\mathrm{UKIRT}}-0.018(J-K)_{\mathrm{UKIRT}}, \\
J-K & =0.936(J-K)_{\mathrm{UKIRT}}, \\
H-K & =0.960(H-K)_{\mathrm{UKIRT}},
\end{aligned}
$$

given by Casali \& Hawarden (1992). 

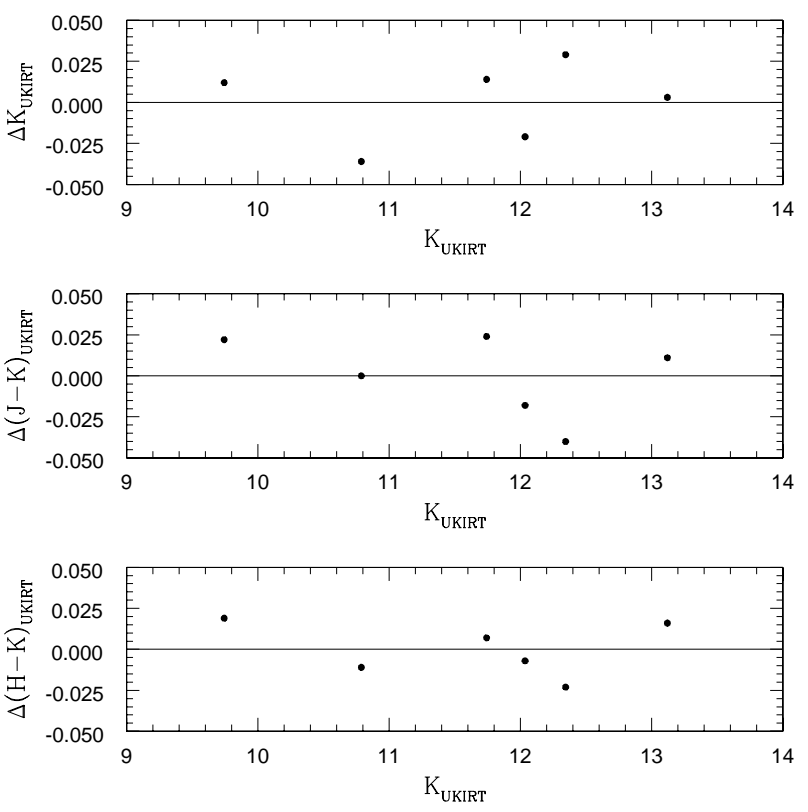

FIG. 4.-Transformation residuals of the UKIRT faint standard stars.

\section{RESULTS AND DISCUSSIONS}

\subsection{Color-Magnitude Diagrams}

Our composite CMDs of Palomar 6 are presented in Figure 5, and the sample CMD data are shown in Table 2. In the table, the positions (cols. [2] and [3]) are presented in pixel units $\left(0\right.$ ". 60 pixel $\left.^{-1}\right)$. The CMD data are also available upon request to the authors. Off-cluster field populations were not obtained during our observation; therefore, the field-star contamination is not removed in our CMDs. Since Palomar 6 lies near the Galactic center, the off-cluster field contamination is expected to be very high (see below) and probably variable because of differential reddening.

Our photometric measurements range from the RGB tip to $\approx 2$ mag below the RHB. The most prominent feature in our CMDs is a well-defined RHB morphology at $H \approx 13.5$ mag and $K \approx 13.3 \mathrm{mag}$. The scatter in the RGB sequences appears to be larger in $J-K$ than in $J-H$. This is most likely due to the bright sky background in the $K$ passband.

TABLE 2

Color-Magnitude Diagram Data

\begin{tabular}{cccrcc}
\hline \hline $\begin{array}{c}\text { ID } \\
(1)\end{array}$ & \multicolumn{1}{c}{$\begin{array}{c}X \\
(2)\end{array}$} & $\begin{array}{c}Y \\
(3)\end{array}$ & \multicolumn{1}{c}{$\begin{array}{c}\text { (4) } \\
\text { (3) }\end{array}$} & $\begin{array}{c}J-K \\
(5)\end{array}$ & $\begin{array}{c}H-K \\
(6)\end{array}$ \\
\hline $47 \ldots \ldots$. & 186.657 & 16.844 & 10.204 & 1.426 & 0.244 \\
$71 \ldots \ldots$. & 237.766 & 25.472 & 10.717 & 1.353 & 0.278 \\
$4 \ldots \ldots \ldots$. & 224.406 & 28.821 & 8.015 & 1.441 & 0.279 \\
$64 \ldots \ldots$. & 196.970 & 46.016 & 10.482 & 1.379 & 0.243 \\
$8 \ldots \ldots \ldots$. & 258.721 & 49.039 & 8.204 & 1.634 & 0.388 \\
$69 \ldots \ldots$. & 29.972 & 52.933 & 10.506 & 1.565 & 0.334 \\
$5 \ldots \ldots \ldots$. & 249.247 & 62.583 & 7.786 & 1.701 & 0.476 \\
$48 \ldots \ldots$. & 165.293 & 69.201 & 10.202 & 1.461 & 0.250 \\
$31 \ldots \ldots$. & 174.410 & 73.902 & 9.614 & 1.462 & 0.257 \\
$1 \ldots \ldots \ldots$. & 221.751 & 80.466 & 7.995 & 1.402 & 0.329 \\
\hline
\end{tabular}

NotE.-Table 2 is presented in its entirety in the electronic edition of the Astronomical Journal. A portion is shown here for guidance regarding its form and content.

\subsection{Comparison with the IR Photometry of Minniti, Olszewski, \& Rieke}

Minniti et al. (1995) obtained $J K$ photometry of Palomar 6 by using the $2.3 \mathrm{~m}$ telescope equipped with a $256 \times 256$ $\mathrm{HgCdTe}$ NICMOS3 array at the Steward Observatory. Their image scale was the same as ours $\left(0.60\right.$ pixel $\left.^{-1}\right)$, but their observations covered wider area $(6 ! 6 \times 2 ! 5)$. To calibrate their photometry, they observed the standard stars of Elias et al. (1982). Since standard stars of Elias et al. are too bright for their telescope and camera configuration, they defocused the telescope to prevent the saturation of bright stars on the detector. They also observed the globular cluster M22 during the same night, and they made comparisons with that of Frogel, Persson, \& Cohen (1983),

$$
\begin{gathered}
\Delta K_{\mathrm{M}-\mathrm{F}}=-0.15 \pm 0.11 \text { for } n=3, \\
\Delta J_{\mathrm{M}-\mathrm{F}}=0.19 \pm 0.15 \text { for } n=4,
\end{gathered}
$$

where $\Delta K_{\mathrm{M}-\mathrm{F}}$ and $\Delta J_{\mathrm{M}-\mathrm{F}}$ refer to Minniti et al. minus Frogel et al. (The error is $1 \sigma$ level.) They noted that the differences are large because their common stars are typically bright and close to the nonlinear regime of the detector array.

We compare our photometry with that of Minniti et al. in Figure 6 . The differences are in the sense our photometry minus that of Minniti et al. We compared 134 common bright stars ( $K \leq 12 \mathrm{mag}$ in our magnitude scale) and obtained unexpected results.

1. On average, our $K$ magnitudes are $1.384 \pm 0.024$ mag brighter than those of Minniti et al. (The error is that of the mean.) There exists a gradient in the difference with a slope of $\Delta K \propto 0.099 K$, in the sense that our $K$ magnitudes are slightly brighter than the mean value for the bright stars.

2. On average, our $J$ magnitudes are $0.705 \pm 0.021 \mathrm{mag}$ brighter than those of Minniti et al. There also exists a gradient in the difference with a slope of $\Delta J \propto 0.108 J$, in the sense that our $J$ magnitudes for the bright stars are slightly brighter than the mean value.

3. Our $J-K$ colors are $0.679 \pm 0.017$ mag redder than those of Minniti et al.

The discrepancies between our work and Minniti et al. are too large to be fully explained by the differences between Minniti et al. and Frogel et al. in M22. We also show CMDs by using stars common to our work and that of Minniti et al. Figure 7 (left) represents our photometry, and Figure 7 (right) represents that of Minniti et al. ${ }^{7}$ See also Table 3.

The off-cluster field-star contamination is expected to be very high toward Palomar 6, as discussed in the previous section, and it is necessary to know the membership of RGB stars in our study. On 1988 May 23, we obtained high-resolution $(\lambda / \Delta \lambda \geq 40,000)$ IR echelle spectra of seven bright RGB stars in Palomar 6 and Arcturus for the ${ }^{12} \mathrm{CO}(2-0)$ band head centered at $\approx 22045 \AA$ by using the $3.5 \mathrm{~m}$ NASA Infrared Telescope Facilities. To derive the heliocentric radial velocities, we cross-correlated our target spectra with

\footnotetext{
${ }^{7}$ D. Minniti kindly provided his table for Palomar 6. Part of his table is shown in Table 3 . His $J-K$ color values in col. (6) are 0.6 mag larger than those from col. (5) minus col. (4). In Fig. 7, we adopted the color from col. (5) minus col. (4). Note that Minniti et al. (1995) used the color in col. (6). in their Fig. 3.
} 

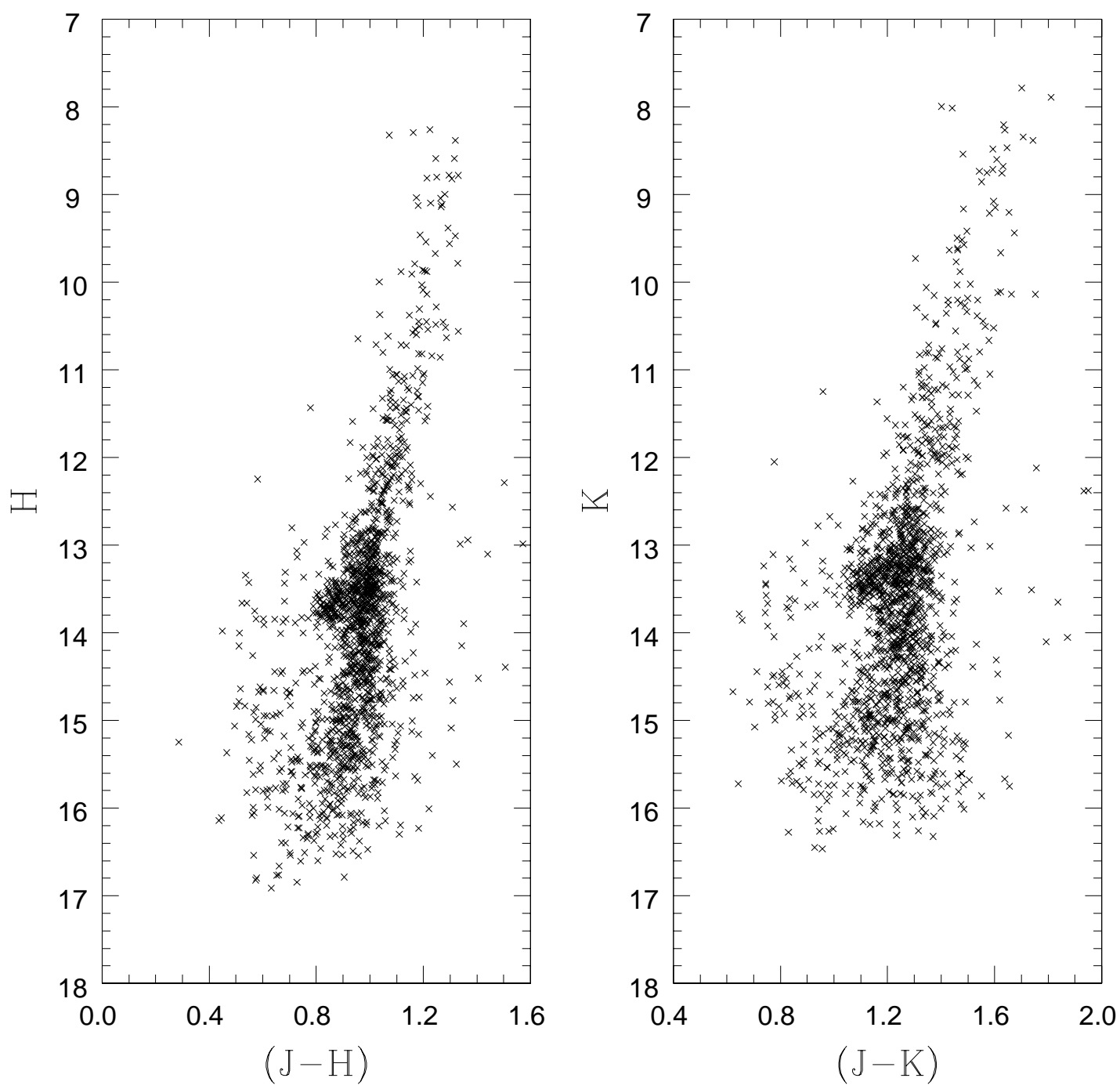

FIG. 5.-Composite IR CMDs of Palomar 6

that of Arcturus ( $v_{r}=-5.2 \mathrm{~km} \mathrm{~s}^{-1}$; Evans 1967). Table 4 shows our heliocentric radial velocity measurements for Palomar 6 RGB stars (see also Fig. 3). Column (3) of the table shows the membership of RGB stars based on their heliocentric radial velocities. (Hereafter, we refer to stars A, $\mathrm{C}, \mathrm{D}$, and $\mathrm{G}$ as membership RGB stars.) It should be noted that the previous radial velocity of Palomar 6 is based on the low-resolution spectroscopic study of Minniti (1995), in which the internal measurement error is much larger than that in ours. We will discuss the spectroscopic study of Palomar 6 RGB stars in a forthcoming paper (Lee, Carney, \& Balachandran 2002).

TABLE 3

Palomar 6 Data From Minniti

\begin{tabular}{cccccccc}
\hline \hline $\begin{array}{c}X \\
(1)\end{array}$ & $Y$ & $r$ & $K$ & $J$ & $J-K$ & $\sigma K$ & $\sigma J$ \\
$(2)$ & $(3)$ & $(4)$ & $(5)$ & $(6)$ & $(7)$ & $(8)$ \\
\hline $190.8 \ldots \ldots$ & 321.0 & 1.30 & 10.65 & 11.47 & 1.42 & 0.01 & 0.01 \\
$194.2 \ldots \ldots$. & 323.6 & 5.60 & 12.54 & 13.62 & 1.68 & 0.04 & 0.04 \\
$182.5 \ldots \ldots$. & 315.5 & 8.70 & 13.92 & 14.94 & 1.62 & 0.11 & 0.13 \\
$189.0 \ldots \ldots$ & 310.9 & 9.20 & 10.05 & 11.04 & 1.59 & 0.01 & 0.01 \\
\hline
\end{tabular}

In Figure 8, we show $V J H K$ multicolor CMDs for Palomar 6, combining our $J H K$ photometry and $V$ photometry of Ortolani et al. (1995). Since we already knew the four cluster membership RGB stars (filled circles), we drew RGB/RHB fiducial sequences with arbitrary widths in the $(V-K, V) \mathrm{CMD}$ by eye in the extension of the four member-

TABLE 4

Heliocentric Radial Velocities of Palomar 6 RGB Stars

\begin{tabular}{|c|c|c|}
\hline $\begin{array}{l}\mathrm{ID}^{1} \\
(1)\end{array}$ & $\begin{array}{c}v_{r} \\
\left(\mathrm{~km} \mathrm{~s}^{-1}\right) \\
(2)\end{array}$ & $\begin{array}{c}\text { Membership } \\
\text { (3) }\end{array}$ \\
\hline A................. & 185.3 & Yes \\
\hline B ................ & 26.7 & No \\
\hline $\mathrm{C} \ldots \ldots \ldots \ldots \ldots$ & 173.5 & Yes \\
\hline $\mathrm{D}, \ldots \ldots \ldots \ldots \ldots$ & 186.8 & Yes \\
\hline E ................ & -13.5 & No \\
\hline F ................... & 134.5 & No \\
\hline $\mathrm{G} \ldots \ldots \ldots \ldots \ldots$ & 176.7 & Yes \\
\hline Mean........ & \multicolumn{2}{|c|}{$180.6 \pm 6.5$} \\
\hline
\end{tabular}

${ }^{1}$ See also Fig. 3. 

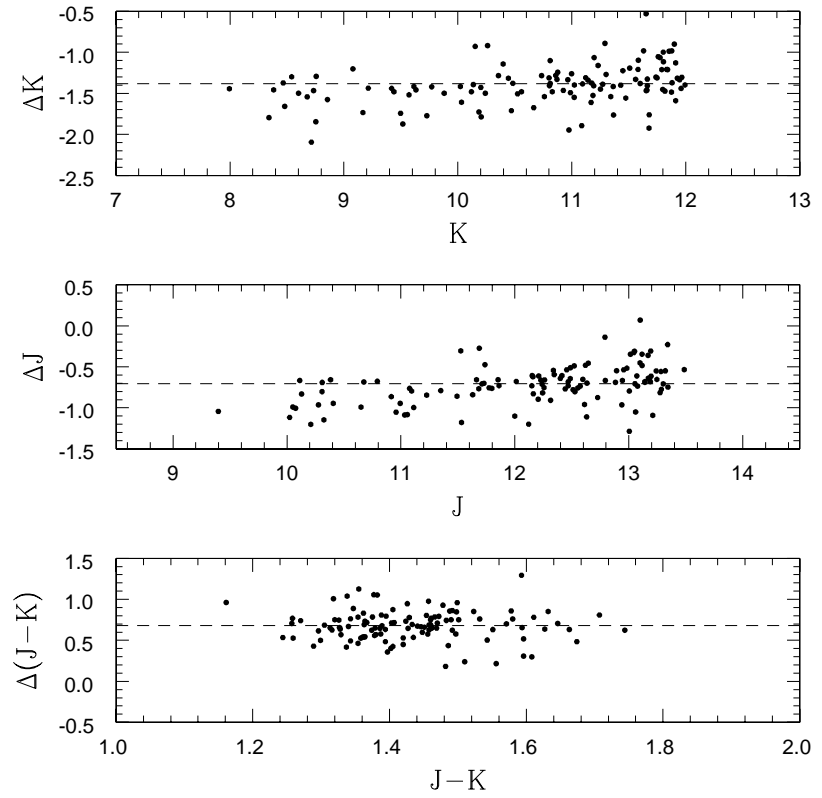

FIG. 6.-Residuals of the photometry between our work and Minniti, Olszewski, \& Rieke (1995). The differences are in the sense our photometry minus that of Minniti et al. ship RGB stars. We then removed outliers by cross-examining $H-K$ and $J-K$ colors. The remaining stars (likely the cluster membership stars) are plotted as crosses in the figure. Our $(V-K, K)$ and $(V-K, V)$ CMDs may show that our $J H K$ photometry in Figure 5 suffers serious field-star contamination, an expected result since Palomar 6 lies near the Galactic center. The RGB stars are clearly separated into at least three distinctive branches in $(V-K, K)$ and $(V-K, V)$ CMDs, in which the cluster's RGB stars are located to the blue, indicating that Palomar 6 is more metal-poor or less heavily reddened than the surrounding fields.

In Figure 9, we show $(V-I, V)$ CMDs for 47 Tuc (Kaluzny et al. 1998) and Palomar 6 (Ortolani et al. 1995). For the 47 Tuc CMD, we show a model isochrone for $[\mathrm{Fe} / \mathrm{H}]=-0.83$ and $[\alpha / \mathrm{Fe}]=+0.30$ (Bergbusch \& VandenBerg 2001) and the location of RHB stars in 47 Tuc (see also Fig. 34 of VandenBerg 2000). For the Palomar 6 CMD, circles represent Palomar 6 membership RGB stars, crosses represent stars selected from multicolor CMDs (see Fig. 8), and gray dots represent stars within $1^{\prime}$ from the cluster center. Since the model isochrone provides an excellent match with 47 Tuc, we adopt this model isochrone as a fiducial sequence for 47 Tuc. We then derived the relative distance modulus and interstellar reddening for Palomar 6 with respect to those for 47 Tuc by adjusting this model isochrone's magnitude to match those of four Palomar 6 RGB stars (circles) and adjusting the model isochrone's color to

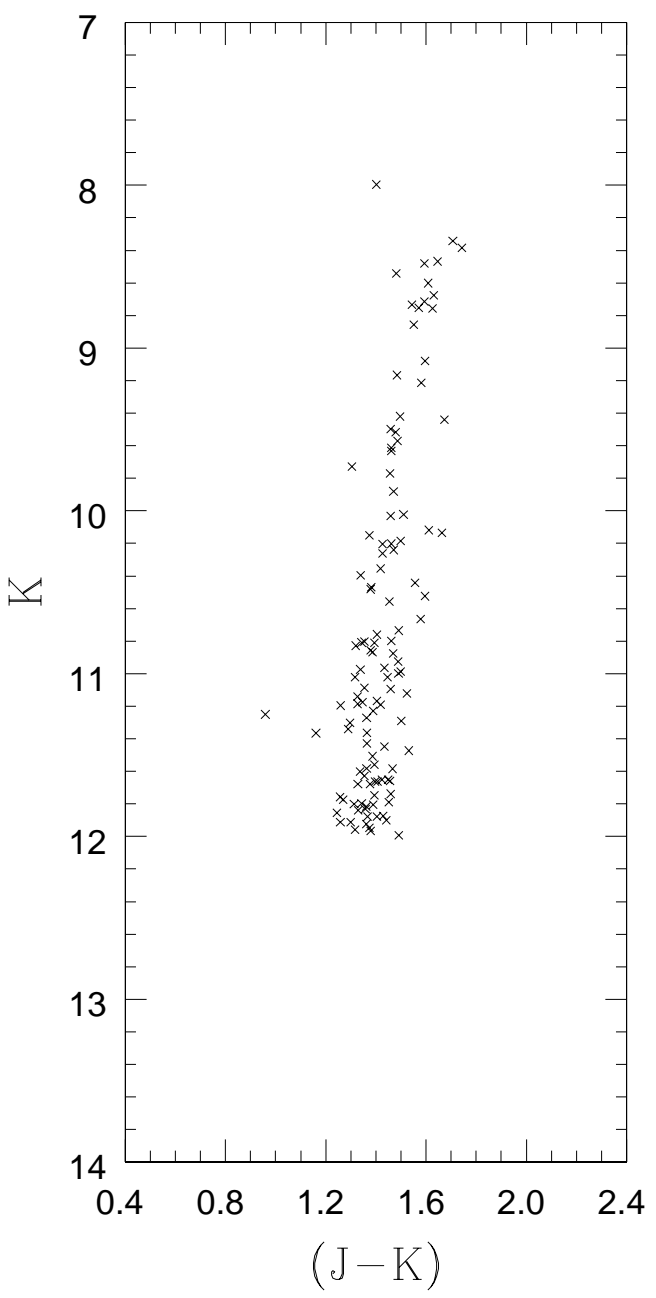

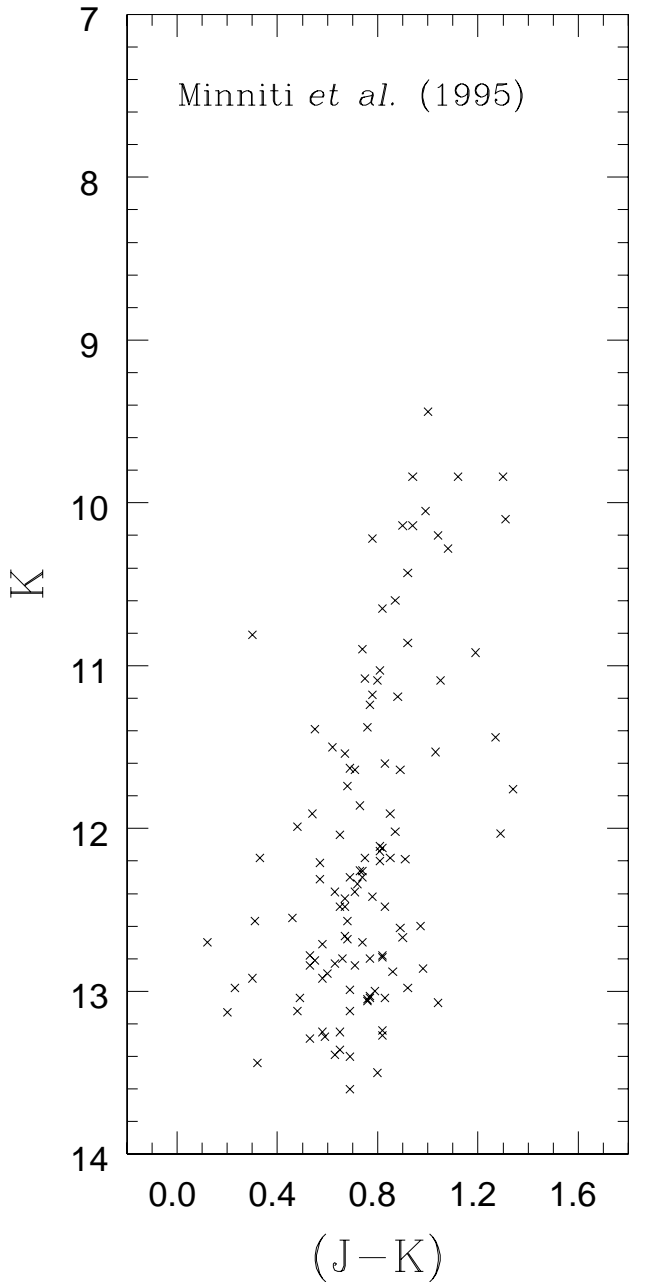

FIG. 7.-Comparison of CMDs by using stars common to our work (left) and that of Minniti et al (right) 

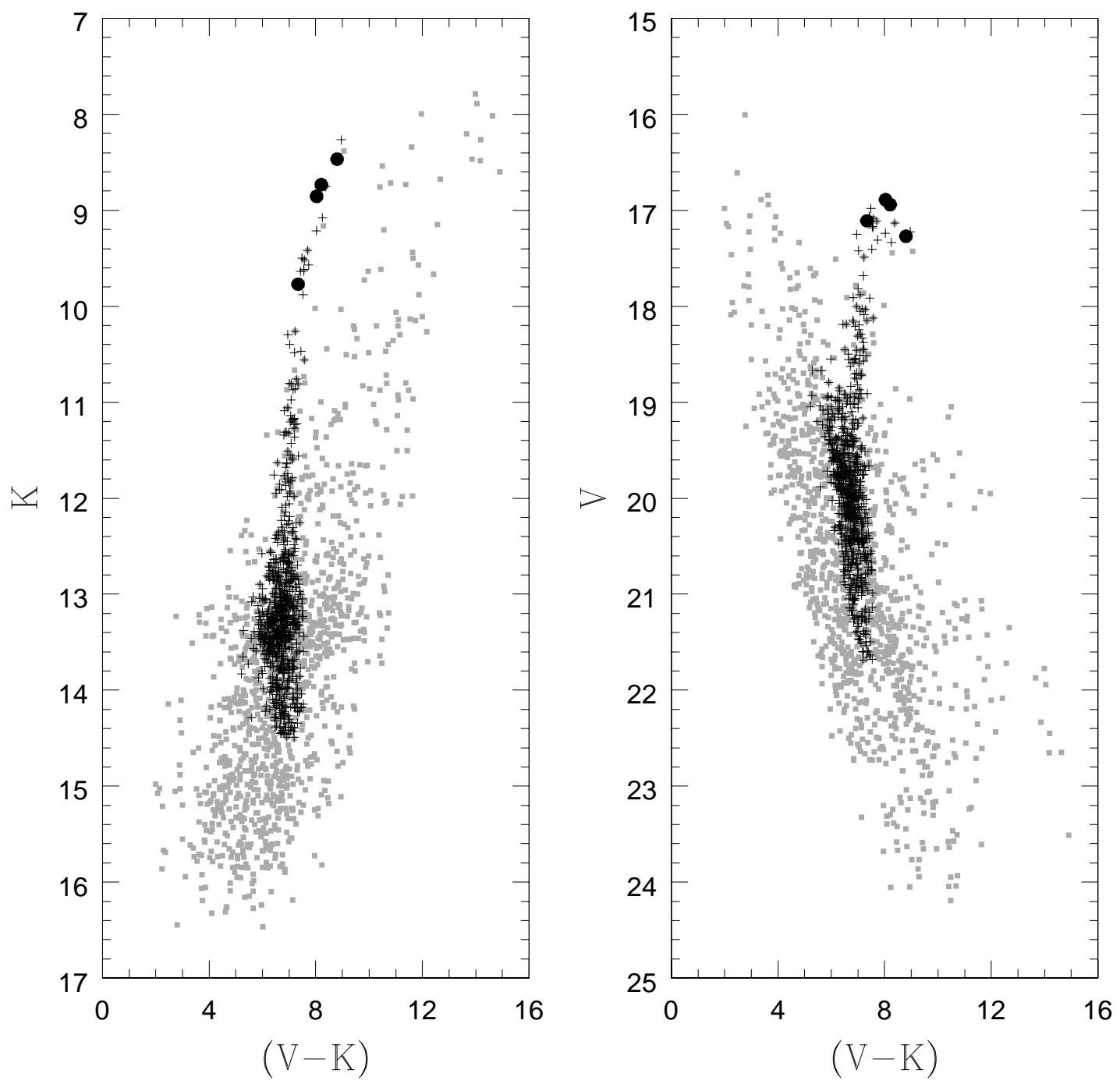

FIG. 8.-VJHK multicolor CMDs of Palomar 6 and possible membership stars, showing membership stars confirmed by radial velocity measurements (circles) and possible membership stars (crosses).

match the color that Ortolani et al. (1995) obtained at the intersection of the RHB and the RGB. The crossing point of the two dashed lines centered at $V=19.7 \mathrm{mag}$ and $V-I=2.7$ mag indicates the RHB magnitude obtained by Ortolani et al. (1995). The color difference $\Delta(V-I)=1.65$ mag corresponds to $\Delta E(B-V)=1.27$ mag, assuming $E(V-I)=1.3 E(B-V)$, and the magnitude difference $\Delta V=5.1 \mathrm{mag}$ corresponds to $\Delta(m-M)_{0}=1.16 \mathrm{mag}$, in the sense of Palomar 6 minus 47 Tuc. It should be noted that the RHB magnitude reported by Ortolani et al. (1995) appears to be $\approx 0.4-0.5 \mathrm{mag}$ fainter than that expected from 47 Tuc (the closed box at $V \approx 19.2$ ). Using these relative distance modulus and interstellar reddening values, we compare $J K$ photometry for 47 Tuc and Palomar 6 in Figure 10. The circles represent the 47 Tuc RGB/RHB stars (Frogel, Persson, \& Cohen 1981), and dotted lines at $K \approx 6.5 \mathrm{mag}$ represent the RGB tip $K$ magnitude for 47 Tuc (Ferraro et al. 2000). The colors and the magnitudes of the current work in $(a)$ and those of Minniti et al. in (b) are shifted by $\Delta(J-K)=-0.67 \mathrm{mag}$ and $\Delta K=1.60 \mathrm{mag}$, assuming $\Delta E(B-V)=1.27$ and $\Delta(m-M)_{0}=1.16$ mag between Palomar 6 and 47 Tuc. In Figure 10a, we also show the mean magnitude and color of the Palomar 6 RHB stars (see below). Our RGB tip $K$ magnitude appears to be consistent with that of 47 Tuc, while that of Minniti et al. (1995) appears to be $\approx 1.5 \mathrm{mag}$ fainter than that of 47 Tuc.

A comparison with the Two Micron All-Sky Survey (2MASS; Cutri et al. 2000) may also provide an opportunity to assess our photometry, although 2MASS may have a problem in crowded fields due to a larger pixel scale (2" pixel $^{-1}$ ). Figure 11 shows a comparison of our photometry with that of 2MASS. Since 2MASS employed the $K_{S}$ passband, we converted our magnitudes and colors into those of the 2MASS photometric system by using the transformation relations given by Carpenter (2001). The results are

$$
\begin{aligned}
\Delta K_{S} & =-0.010 \pm 0.021, \\
\Delta\left(J-K_{S}\right) & =-0.152 \pm 0.018, \\
\Delta\left(H-K_{S}\right) & =-0.070 \pm 0.020,
\end{aligned}
$$

where the differences are in the sense our work minus 2MASS in the 2MASS photometric system. (The errors are those of the mean.) The difference in the $J-K_{S}$ color is rather large, in the sense that our photometry is $\approx 0.15 \mathrm{mag}$ bluer than that of 2MASS, but the differences are much 

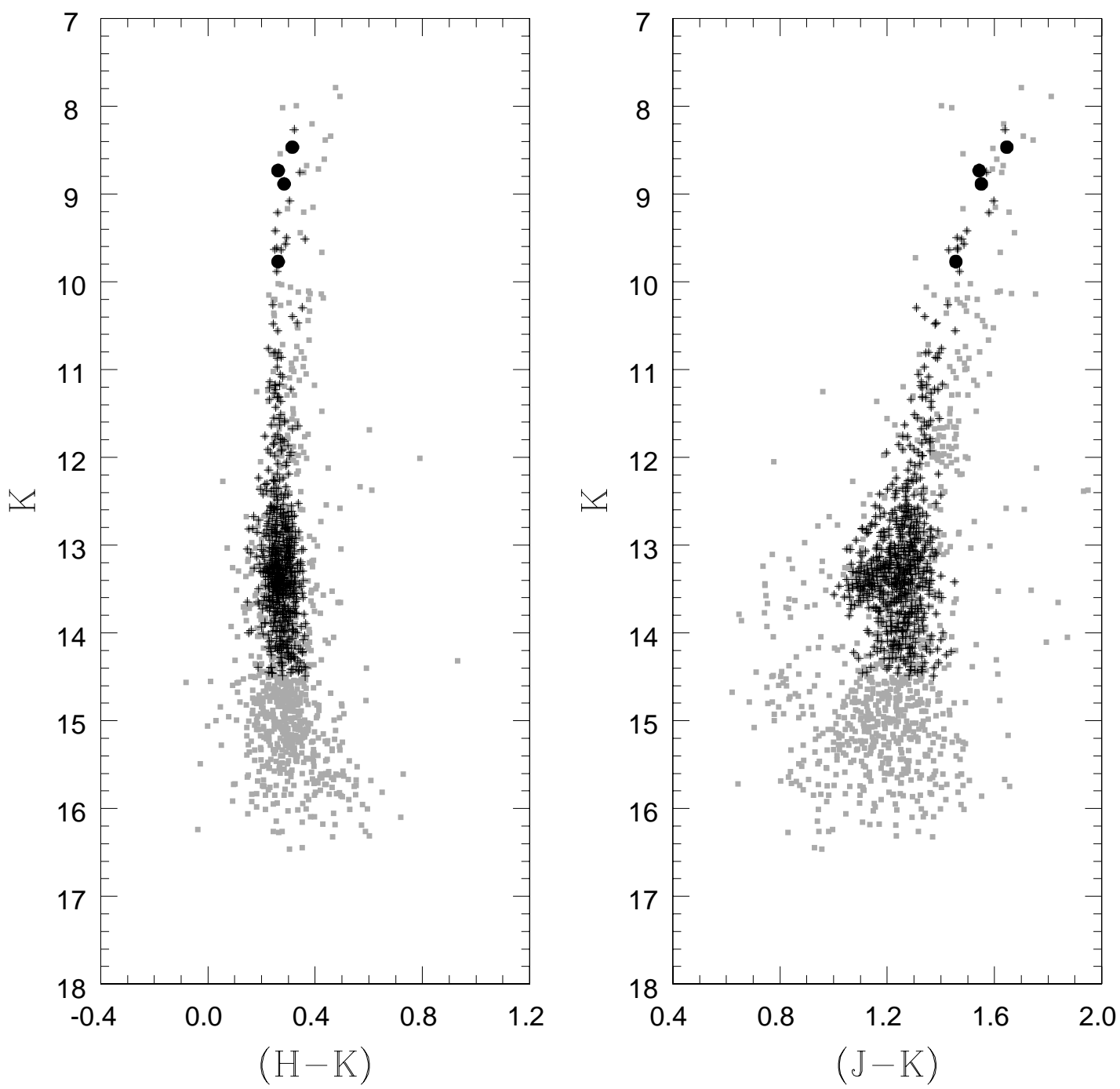

FIG. 8.-Continued

smaller than 1.4 mag in the $K_{S}$ passband or 0.7 mag in the $J-K_{S}$ color.

Finally, we observed the four high-proper-motion stars (LHS 514, 2584, 2824, and 3094) during the same night. Table 5 shows a comparison of the unpublished results of Carney and our measurements. As can be seen in the table, our measurements are in good agreement with that of Carney to within $0.1 \mathrm{mag}$. Therefore we conclude that the discrepancy between our work and that of Minniti et al. (1995) is most likely due to the inaccurate photometric measurement by Minniti et al. (1995).

\subsection{Distance Modulus and Interstellar Reddening}

We derive the distance modulus and the interstellar reddening for Palomar 6 by using the method described by Kuchinski et al. (1995), who recommended using the magnitude and the color at the intersection of the RGB and the RHB in IR photometry. In Figure 12, we show the $J K$ CMD for 47 Tuc of Frogel et al. (1981). We also show the slope of the RGB and the mean RHB magnitude and color. Kuchinski et al. (1995) obtained $(J-K)[\mathrm{RGB}, \mathrm{RHB}]=0.55$ mag and $K[\mathrm{RGB}, \mathrm{RHB}]=12.02 \mathrm{mag}$ at the intersection of the RGB and RHB for 47 Tuc. Following a similar method, we derived these values for Palomar 6, using the possible membership stars obtained from Figure 8. First, we derive the slope of the Palomar 6 RGB stars,

$$
J-K=-(0.0751 \pm 0.0040) K+(2.2030 \pm 0.0452) \text {. }
$$

We then define a rectangle whose sides are parallel to $J-K$ and $K$ by eye and calculate the mean color and magnitude. We obtain $\langle J-K\rangle_{\mathrm{RHB}}=1.09 \pm 0.04 \mathrm{mag}$ and $\langle K\rangle_{\mathrm{RHB}}=13.53 \pm 0.15 \mathrm{mag}$ for Palomar 6. (The error is $1 \sigma$ level.) In Figure 12, we show the slope of the RGB, the rectangle that we adopted, and the mean RHB color and
TABLE 5

Comparisons of $K$ Magnitudes of High-Proper-Motion Stars

\begin{tabular}{|c|c|c|}
\hline ID & Carney & This Work \\
\hline LHS $514 \ldots . . . . .$. & 9.19 & $9.174 \pm 0.011$ \\
\hline LHS $2584 \ldots \ldots .$. & 12.38 & $12.342 \pm 0.012$ \\
\hline LHS $2824 \ldots \ldots .$. & 9.75 & $9.841 \pm 0.011$ \\
\hline LHS $3094 \ldots \ldots .$. & 12.25 & $12.216 \pm 0.017$ \\
\hline
\end{tabular}




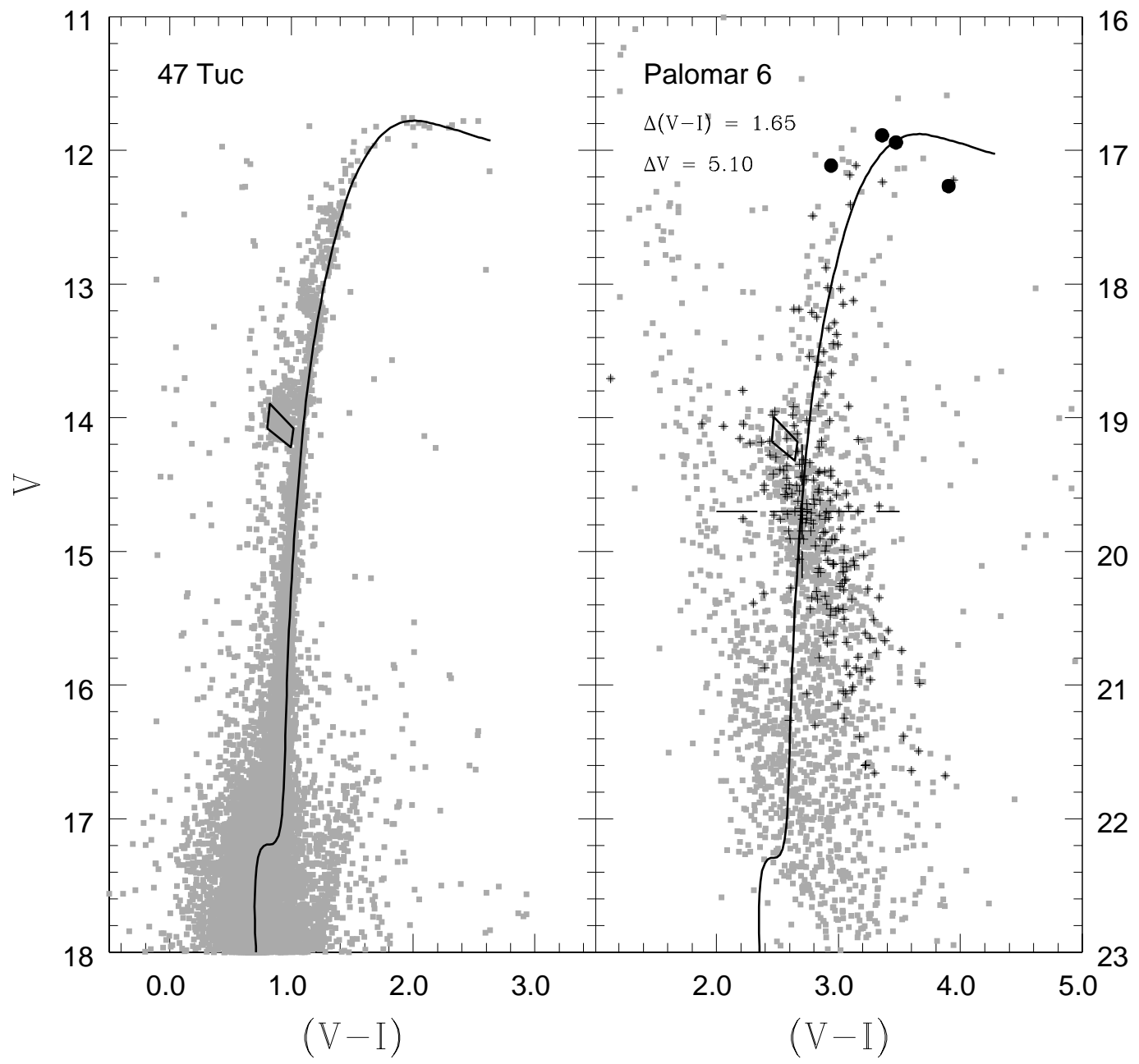

FIg. 9.- VI CMDs for 47 Tuc (Kaluzny et al. 1998) and Palomar 6 (Ortolani, Bica, \& Barbuy 1995), showing a model isochrone for [Fe/H] $=-0.83$ and $[\alpha / \mathrm{Fe}]=+0.30$ (Bergbusch \& VandenBerg 2001) and the location of RHB stars in 47 Tuc. Using this model isochrone, we derived the relative distance modulus and interstellar reddening for Palomar 6 with respect to those for 47 Tuc. Right, Palomar 6 membership RGB stars confirmed by radial velocity measurements (circles), stars selected by using multicolor CMDs (crosses; see Fig. 8), stars within $1^{\prime}$ from the cluster center (gray dots), and the crossing point of the two dashed lines representing the RHB magnitude by Ortolani et al. (1995). The color difference $\Delta(V-I)=1.65$ corresponds to $\Delta E(B-V)=1.27$, assuming $E(V-I)=1.3 E(B-V)$, and the magnitude difference $\Delta V=5.1$ mag corresponds to $\Delta(m-M)_{0}=1.19$ mag, in the sense of Palomar 6 minus 47 Tuc.

magnitude for Palomar 6. We obtain $(J-K)[\mathrm{RGB}, \mathrm{RHB}]=$ 1.19 mag for Palomar 6 by using equation (6).

By comparing the magnitudes $K[\mathrm{RGB}, \mathrm{RHB}]$ and the colors $(J-K)[\mathrm{RGB}, \mathrm{RHB}]$ at the RGB and RHB intersection points of 47 Tuc and Palomar 6, we obtained $\Delta E(B-V)=1.21 \mathrm{mag}$ and $\Delta(m-M)_{0}=1.09 \mathrm{mag}$, assuming $E(J-K)=0.53 E(B-V)$ and $A_{K}=0.35 E(B-V)$ (Rieke \& Lebofsky 1985). Using the distance modulus $(m-M)_{0}=13.25 \mathrm{mag}$ and the interstellar reddening $E(B-V)=0.04$ mag for 47 Tuc (Harris 1996), we obtained the interstellar reddening $E(B-V)=1.25 \mathrm{mag}$ and the distance modulus $(m-M)_{0}=14.34$ mag for Palomar 6 . We also derive these values using the $J H$ CMD. We obtained $(J-H)[\mathrm{RGB}, \mathrm{RHB}]=0.52 \mathrm{mag}$ and $H[\mathrm{RGB}$, $\mathrm{RHB}]=12.10 \mathrm{mag}$ for 47 Tuc and $(J-H)[\mathrm{RGB}$, $\mathrm{RHB}]=0.95 \mathrm{mag}$ and $H[\mathrm{RGB}, \mathrm{RHB}]=13.77 \mathrm{mag}$ for Palomar 6. Assuming $E(J-H)=0.33 E(B-V)$ and $A_{H}=0.54 E(B-V)$ (Rieke \& Lebofsky 1985), we obtained $E(B-V)=1.34$ mag and $(m-M)_{0}=14.22$ mag for Palomar 6. For our study, we adopt the mean values $E(B-V)=1.30 \mathrm{mag}$ and $(m-M)_{0}=14.28 \mathrm{mag}$ for Palomar 6. Our interstellar reddening estimate for Palomar 6 shows good agreement with previous values. However, our distance modulus estimate is $\approx 0.5 \mathrm{mag}$ smaller than that of Ortolani et al. (1995), who obtained $(m-M)_{0}=14.76$ by using the HB magnitude shown in Figure 8. Using our distance modulus, the distance of Palomar 6 from the sun is $\approx 7.2 \mathrm{kpc}$. The Galactocentric distance $R_{\mathrm{GC}}$ becomes $0.9 \mathrm{kpc}$ if $R_{0}=8.0 \mathrm{kpc}$ (Reid 1993). Palomar 6 is clearly located in the Galaxy's central regions.

Finally, a caution is advised for using RHB magnitudes to estimate the distance modulus of the metal-rich globular clusters. Sandage (1990) studied the vertical height of the horizontal branch of the globular clusters as a function of metallicity. His study suggested that the vertical height of the HB increases with metallicity. For example, the vertical height of the 47 Tuc RHB is $\approx 0.7$ mag in the visual passband. Palomar 6 is expected to have the similar value of the RHB vertical height (see his Fig. 16). In our CMDs, the Palomar 6 RHB vertical height is $\approx 0.5-0.6$ mag in the $K$ passband. It should also be noted that the $K_{0}$ magnitude of field red clump stars in the Galactic bulge is $\approx 13$ mag (Alves 2000), having magnitude similar to Palomar 6 RHB stars, depending on the interstellar reddening toward field red 


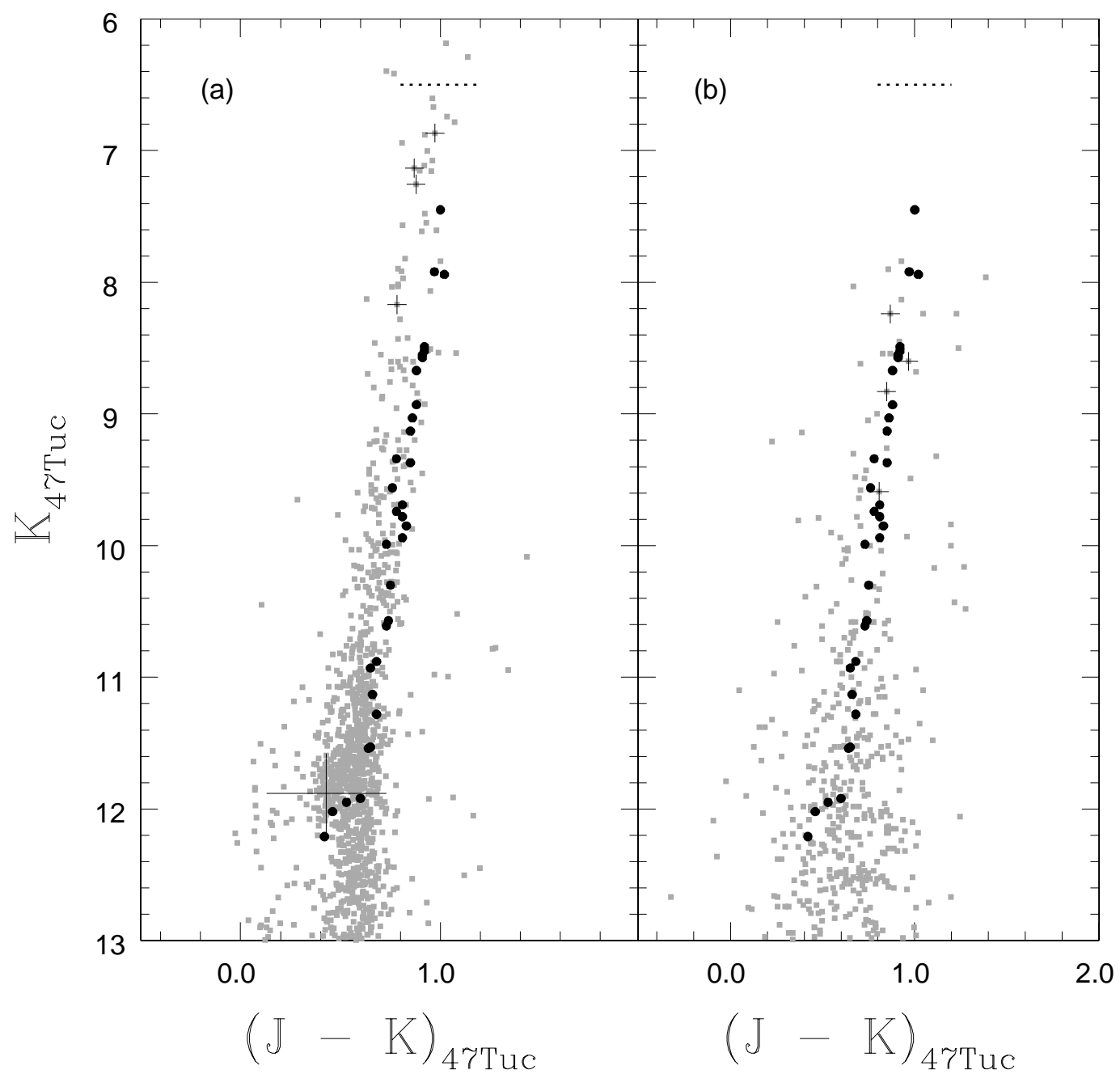

FIG. 10.-Comparison of $J K$ photometry of Palomar 6 with that of 47 Tuc, showing the 47 Tuc RGB/RHB stars (circles; Frogel, Persson, \& Cohen 1981) and the $K$ magnitude of the brightest RGB stars in 47 Tuc (dotted lines at $K \approx 6.5 \mathrm{mag}$; Ferraro et al. 2000). The colors and the magnitudes of the current work in $(a)$ and those of Minniti et al. in $(b)$ are shifted by $\Delta(J-K)=-0.67$ mag and $\Delta K=1.60$ mag, assuming $\Delta E(B-V)=1.27$ and $\Delta(m-M)_{0}=1.16$ mag between Palomar 6 and 47 Tuc. In (a), we show the mean magnitude and color of the Palomar 6 RHB stars. The four membership RGB stars are represented by crosses in each panel. Our RGB tip $K$ magnitude is consistent with that of 47 Tuc, while that of Minniti et al. is $\approx 1.5$ mag fainter than that of 47 Tuc.

clump stars. Thus accidental inclusion of field red clump stars in our Palomar 6 CMD could be high, and future photometric study of the off-cluster field would be desirable.

\subsection{Metallicity}

Kuchinski et al. (1995) studied the slope of the RGB in the $(J-K, K) \mathrm{CMD}$ as a reddening-and distance-independent metallicity index for metal-rich globular cluster systems. They derived a linear relation between metallicity and the slope of the RGB,

$$
[\mathrm{Fe} / \mathrm{H}]=-(3.09 \pm 0.90)-(24.85 \pm 8.90)(\mathrm{RGB} \text { slope }) \text {, }
$$

where the slope of the RGB is defined as $J-K \propto K(\mathrm{RGB}$ slope). Kuchinski \& Frogel (1995) recalculated this linear relation with the addition of two more clusters, and they obtained

$$
[\mathrm{Fe} / \mathrm{H}]=-(2.98 \pm 0.70)-(23.84 \pm 6.83)(\mathrm{RGB} \text { slope }) \text {. }
$$

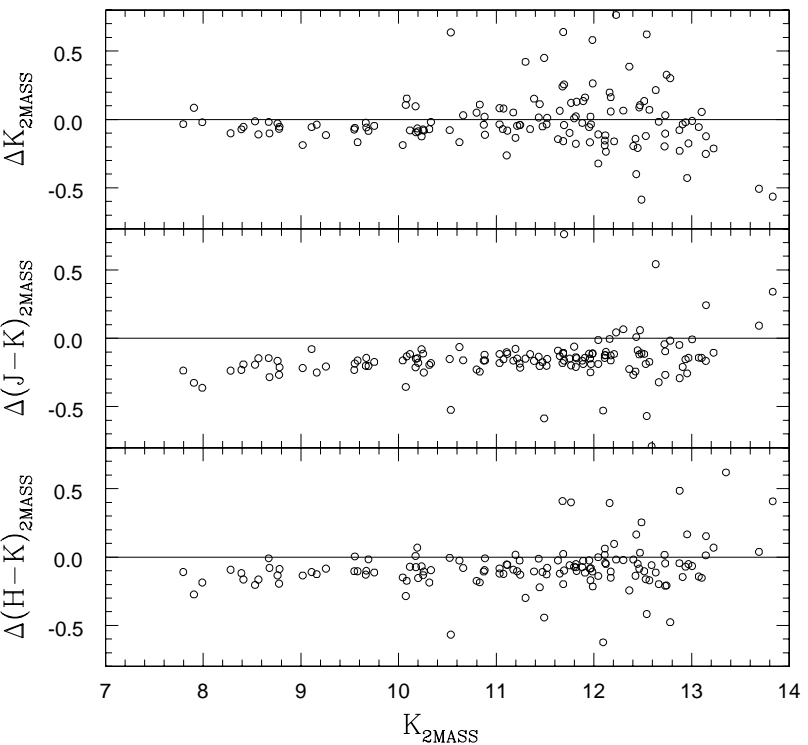

FIG. 11.-Comparison with 2MASS photometry. The differences are in the sense our photometry minus that of 2MASS. 


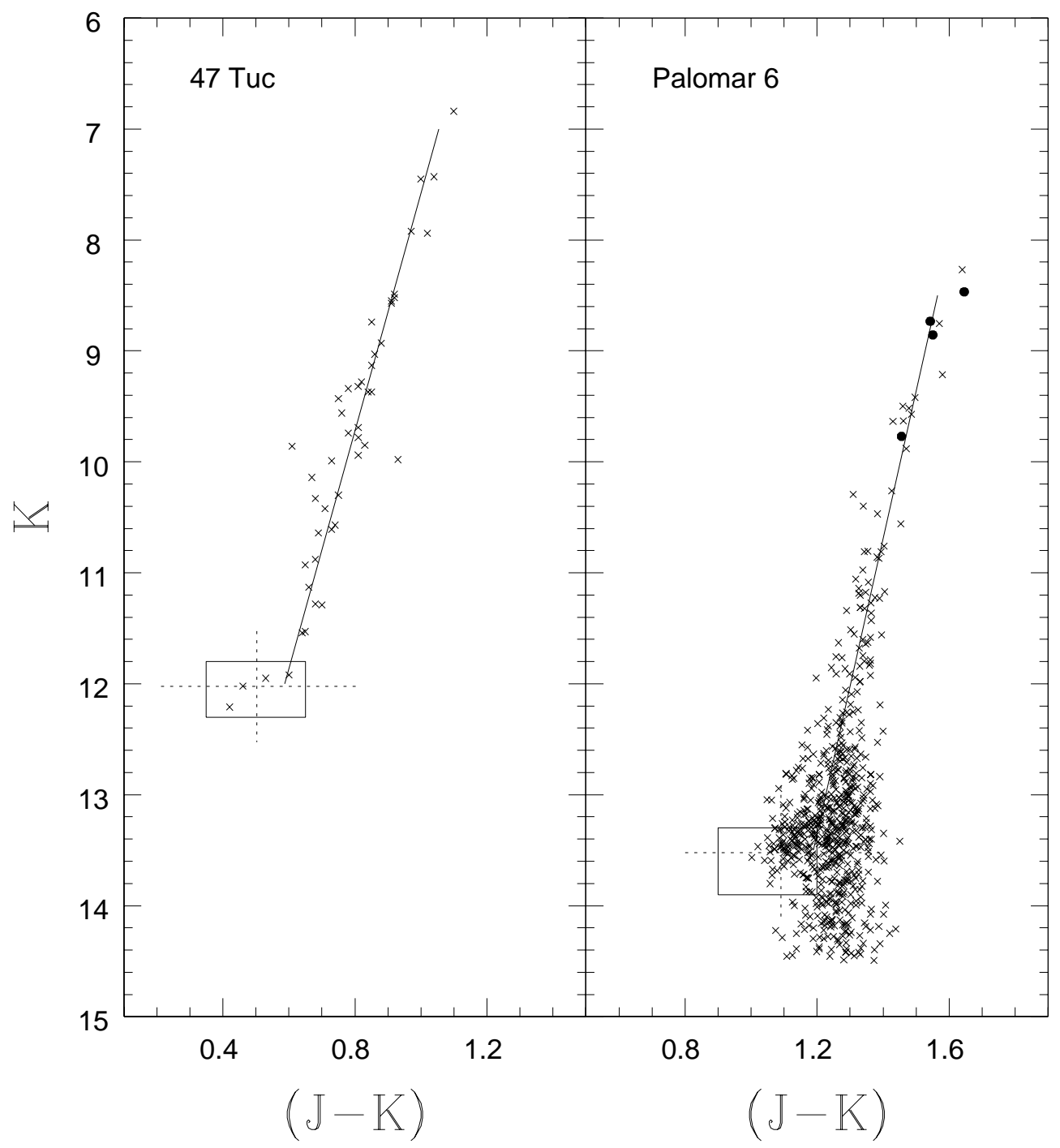

FIg. 12.-Mean RHB colors and magnitudes for Palomar 6 RHB and 47 Tuc. The center of the dotted lines represents the mean RHB color and magnitude, using stars inside the rectangle. The filled circles represent the Palomar 6 RGB membership stars.

We derived the slope of RGB of Palomar 6 and our result is shown in Figure 12 and equation (6). As Kuchinski et al. (1995) and Kuchinski \& Frogel (1995) noted, the slope of the RGB does not depend on the interstellar reddening, and therefore, conversion to dereddened colors and magnitudes is not necessary. With our slope for Palomar 6, we obtained $[\mathrm{Fe} / \mathrm{H}]=-1.22 \pm 0.18$ by using equation (7) (Kuchinski et al. 1995) and $-1.19 \pm 0.18$ by using equation (8) (Kuchinski \& Frogel 1995). It should be noted that our results are from selected Palomar 6 RGB stars as discussed in the previous section. If we use all RGB stars, $[\mathrm{Fe} / \mathrm{H}]=-1.05 \pm 0.19$ by using equation (7) and $-1.03 \pm 0.19$ by using equation (8), and they are slightly lower than those from selected RGB stars. ${ }^{8}$

Our metallicity estimate for Palomar 6 is consistent with that of Malkan (1981), but it does not agree with those of

\footnotetext{
${ }^{8}$ Referee kindly pointed out that the IR HB morphology of Palomar 6 may indicate that its metallicity is between $\mathrm{M} 69([\mathrm{Fe} / \mathrm{H}]=-0.6)$ and NGC $6553([\mathrm{Fe} / \mathrm{H}]=-0.3)$ from Fig. 1 of Ferraro et al. (2000). It should be noted, however, that the HB morphology is vulnerable to other effects besides metallicity (see for example Lee, Demarque, \& Zinn 1994).
}

Zinn (1985), Ortolani et al. (1995), and Minniti (1995). The metallicity of Palomar 6 will be discussed in the subsequent paper. We obtained $[\mathrm{Fe} / \mathrm{H}]$ of the three RGB stars in Palomar 6 by using high-resolution $(\lambda / \Delta \lambda \geq 40,000)$ IR echelle spectra. Our metallicity of Palomar 6 by using IR spectra is $[\mathrm{Fe} / \mathrm{H}]=-1.08 \pm 0.06$, consistent with those derived from the slope of the RGB.

\section{SUMMARY}

We have presented the $J H K$ photometry of Palomar 6 . Our photometric measurements range from the RGB tip to $\approx 2$ mag below the RHB. Our photometry does not agree with that of Minniti et al. (1995) and an independent study using the southern telescope facilities would be very desirable in the future.

We have discussed the distance modulus and interstellar reddening of Palomar 6 by comparing the mean magnitude and color of the RHB stars with those of 47 Tuc. Our interstellar reddening estimate is consistent with previous values obtained by others, while our distance modulus is slightly smaller than that of Ortolani et al. (1995), who obtained the 
distance modulus of Palomar 6 by using a similar method in the visual passband. Nevertheless, our study has suggested that Palomar 6 is clearly located in the Galaxy's central regions.

We have also discussed the metallicity of Palomar 6 by using the slope of the RGB. Our metallicity estimate is in good agreement with that of Malkan (1981), but it does not agree with those of Zinn (1985), Ortolani et al. (1995), and Minniti et al. (1995).

This is part of the Ph.D. thesis work of J.-W. Lee at the University of North Carolina at Chapel Hill. J.-W. Lee thanks Dante Minniti for providing his photometric table. We also thank an anonymous referee for useful comments and a careful review of the paper. This research was supported by the National Aeronautics and Space Administration grant number GO-07318.04-96A from the Space Telescope Science Institute, which is operated by the Association of Universities for Research in Astronomy, Inc., under NASA contract NAS 5-26555 and National Science Foundation grants AST 96-19381 and AST 99-88156. Support for this work was also provided in part by the Creative Research Initiative Program of the Korean Ministry of Science and Technology.
Alves, D. R. 2000, ApJ, 539, 732

Bergbusch. P. A., \& VandenBerg, D. A. 2001, ApJ, 556, 322

Bica, E., Claria, J. J., Piatti, A. E., \& Bonatto, C. 1998, A\&AS, 131, 483

Carpenter, J. M. 2001, AJ, 121, 2851

Casali, M. M., \& Hawarden, T. G. 1992, JCMT-UKIRT Newsl., 4, 33

Cutri, R. M., et al. 2000, 2MASS Second Incremental Data Release (Pasadena: Caltech)

Elias, J. H., Frogel, J. A., Matthews, K., \& Neugebauer, G. 1982, AJ, 87, 1029

Evans, D. S. 1967, in IAU Symp. 30, Determination of Radial Velocities and their Applications, ed. A. H. Batten \& J. F. Heard (New York: Academic Press), 57

Faber, S. M., Friel, E. D., Burstein, D., \& Gaskell, C. M. 1985, ApJS, 57, 711

Ferraro, F. R., Montegriffo, P., Origlia, L., \& Fusi Pecci, F. 2000, AJ, 119 ,

Frogel, J. A., Persson, S. E., \& Cohen, J. G. 1981, ApJ, 246, 842 1983, ApJS, 53, 713

Harris, W. E. 1996, AJ, 112, 1487

Kaluzny, J., Kubiak, M., Szymanski, M., Udalski, A., Krzeminski, W., Mateo, M., \& Stanek, K. Z. 1998, A\&AS, 128, 19

Kuchinski, L. E., \& Frogel, J. A. 1995, AJ, 110, 2844

Kuchinski, L. E., Frogel, J. A., Terndrup, D. M., \& Persson, S. E. 1995, AJ, 109,1131

\section{REFERENCES}

Lee, J.-W., \& Carney, B. W. 1999, AJ, 117, 2868

Lee, J.-W.. Carney, B. W., \& Balachandran, S. C. 2002, in preparation Lee, Y.-W., Demarque, P., \& Zinn, R. 1994, ApJ, 423, 248

Malkan, M. A. 1981, in IAU Colloq. 68, Astrophysical Parameters for Globular Clusters, ed. A. G. Davis Phillip \& D. S. Haynes (Schenectady: L. Davis), 533

Minniti, D. 1995, A\&A, 303, 468

Minniti, D., Olszewski, E. W., \& Rieke, M. 1995, AJ, 110, 1686

Ortolani, S., Bica, E.. \& Barbuy, B. 1995, A\&A, 296, 680

Reid, M. J. 1993, ARA\&A, 31, 345

Rieke, G. H., \& Lebofsky, M. J. 1985, ApJ, 288, 618

Sandage, A. 1990, ApJ, 350, 603

Stetson, P. B. 1987, PASP, 99, 191

. 1990, PASP, 102, 932

1994, PASP, 106, 250

- 1995, DAOPHOTII User's Manual (Victoria: Dominion Astrophys. Obs.)

Turner, A. M. 1995, Cooking with ALLFRAME (Victoria: Dominion Astrophys. Obs.)

VandenBerg, D. A. 2000, ApJS, 129, 315

Zinn, R. 1980, ApJS, 42, 19 .1985, ApJ, 293, 424 